\title{
Neighborhood-Based Descriptors for Porphyrin Dendrimers
}

\author{
Thanga Rajeswari Krishnasamy ${ }^{1}{ }^{(\mathbb{C})}$, Manimaran Angamuthu ${ }^{1, *(\mathbb{C})}$ \\ 1 Department of Mathematics, School of Advanced Sciences, Vellore Institute of Technology, Vellore; \\ shivamayam27@gmail.com (K. T.), marans2011@gmail.com (A. M.); \\ * Correspondence: marans2011@gmail.com;
}

Scopus Author ID 57062968200

Received: 27.08.2021; Revised: 25.10.2021; Accepted: 29.10.2021; Published: 18.11.2021

\begin{abstract}
The symmetry of molecular structures is captured by topological indices, which provide a mathematical vocabulary for predicting features such as boiling temperatures, viscosity, and gyration radius and are also employed in QSPR/QSAR research. Dendrimers are a brand-new type of polymer. It is characterized as a macromolecule due to its highly radiated structure, providing great water solubility and adaptability. Because of these features, dendrimers are a strong alternative for medication delivery. This article investigates some topological indices based on neighborhood degrees such as Modified Randic index, Inverse Sum Index, SK, SK1, and SK2 index for some dendrimers.
\end{abstract}

Keywords: Neighborhood indices; porphyrin dendrimers; poly(propyl) ether imine dendrimer.

(C) 2021 by the authors. This article is an open-access article distributed under the terms and conditions of the Creative Commons Attribution (CC BY) license (https://creativecommons.org/licenses/by/4.0/).

\section{Introduction}

An important use of (connected undirected) graphs is representing an atomic structure by a molecular graph where the node represents atoms, and the edges indicate bondings. This is explored in a discipline called chemical graph theory. The overall topological structure of such a network can be captured in a single number known as a chemical index or a topological index [1-3], which is frequently linked to chemical attributes.

$\mathrm{G}(\mathrm{V}, \mathrm{E})$ is a graph in which $\mathrm{V}$ denotes the set of vertices and $\mathrm{E}$ denotes the set of edges linking the vertices. The degree of a vertex $v$ in graph $G$ is the number of edges incident with $\mathrm{v}$, symbolized by $\operatorname{deg}(\mathrm{v})$. A graph in which each pair of vertices is connected is known as a connected graph. The set of all vertices adjacent to $\mathrm{u}$ is denoted by $\mathrm{N}(\mathrm{u})$ and defined as a Neighborhood set of $u$. The sum degree of vertex $u$ is defined as $S(u)=\sum_{v \in N(u)} \operatorname{deg}(v)[4]$.

Dendrimers are branching polymeric molecules with a high degree of organization. Dendrimers are symmetric about the core, which have a spherical 3D topology. A core, an inner, and an outer shell are the three major components of dendrimers. To regulate features such as solubility, thermal stability, and chemical attachment for different tasks, a dendrimer can be synthesized with varied functionality in each of these components. It has an ancient legacy of use in targeted, ophthalmic, pulmonary, transdermal, and gene-drug delivery [5-10]. Since it is difficult to synthesize dendrimers, they are difficult to produce and expensive to buy. Analyzing topological indices for dendrimers is an effective way to eliminate the cost and timeconsuming laboratory research.

Numerous studies[11-32] have described and determined topological indices of dendrimers like Poly propyl ether imine dendrimer, porphyrin dendrimers, Poly EThylene 
Amido Amine Dendrimer, Phosphorus-Containing Dendrimers, PDI-Cored Dendrimers, Triazine-Based Dendrimers, and Aliphatic Polyamide Dendrimers.

Several research [33-40] have begun to look into Neighborhood-based topological indices. Vignesh Ravi and Kalyani Desikan proposed some Neighborhood-based descriptors, namely $\mathrm{SK}_{\mathrm{N}}, \mathrm{SK} 1_{\mathrm{N}}, \mathrm{SK} 2_{\mathrm{N}}$, Modified Randić index $\left(\mathrm{mR}_{\mathrm{N}}\right)$, and Inverse Sum Index $\left(\mathrm{ISI}_{\mathrm{N}}\right)$ [33]. The indices are given below in equations (1) to (5).

$$
\begin{aligned}
& \operatorname{SK}_{\mathrm{N}}(\mathrm{G})=\sum_{u v \in E}\left[\frac{S(u)+S(v)}{2}\right] \\
& \mathrm{SK} 1_{\mathrm{N}}(\mathrm{G})=\sum_{u v \in E}\left[\frac{S(u) \times S(v)}{2}\right] \\
& \mathrm{SK} 2_{\mathrm{N}}(\mathrm{G})=\sum_{u v \in E}\left(\frac{S(u)+S(v)}{2}\right)^{2} \\
& \operatorname{mR}_{\mathrm{N}}(\mathrm{G})=\sum_{u v \in E}\left(\frac{1}{\max \{S(u), S(v)\}}\right) \\
& \operatorname{ISI}_{\mathrm{N}}(\mathrm{G})=\sum_{u v \in E}\left(\frac{S(u) \mathrm{x} S(v)}{S(u)+S(v)}\right)
\end{aligned}
$$

In this paper, we evaluate the above-mentioned indices for Poly propyl ether imine (PETIM) dendrimer, Zinc porphyrin $\left(\mathrm{DPZ}_{\mathrm{g}}\right)$ dendrimer, and Porphyrin $\left(\mathrm{D}_{\mathrm{g}} \mathrm{P}_{\mathrm{g}}\right)$ dendrimer. We also use MATLAB to depict the 2D representations of the indices.

\section{Materials and Methods}

The Neighborhood degree-based topological indices of dendrimers are our most important computational results. The results were analyzed using the edge partition method and graph-theoretical ideas. In Figures 5-14, the outcomes are visually depicted using MATLAB R2020a.

\section{Results and Discussion}

\subsection{PETIM dendrimer.}

The polynomial of PETIM dendrimer expands from the oxygen as the base and sprouts out at each tertiary nitrogen, which is separated by an eight-bond spacer in every iteration of the dendrimer. Figure 1 shows g growth stages for the molecular graph G of PETIM dendrimer with generation $\mathrm{G}_{\mathrm{g}}$. The PETIM dendrimer graph is composed of four wings and an eightedged central core. In each branch, we have $8+2 \times 8+2^{2} \times 8+\ldots+2^{\mathrm{g}-2} \times 8+4 \times 2^{\mathrm{g}-1}=6 \times 2^{\mathrm{g}}-$ 8 edges. Also, the total number of bonds in $\mathrm{G}$ is $\left(24 \times 2^{\mathrm{g}}\right)-24$. By this, the number of atoms in $\mathrm{G}$ can be given directly as $\left(24 \times 2^{\mathrm{g}}\right)-23$ since it is a tree [11].

The total number of vertices with degree 1 in $\mathrm{G}_{\mathrm{g}}$ is $2^{\mathrm{g}+1}$, which is the number of leaves in the tree. The vertices of degree 3 and degree 2 are $2^{\mathrm{g}+1}-2$ and $20 \times 2^{\mathrm{g}}-21$, respectively.For the pair $(\mathrm{S}(\mathrm{u}), \mathrm{S}(\mathrm{v}))$ where $\mathrm{uv} \in \mathrm{E}(\mathrm{G})$ the edges are partitioned as $2 \times 2^{\mathrm{g}}$ for the pairs $(2,3)$ and $(3,4) ; 8 \times 2^{\mathrm{g}}-12$ for the pair $(4,4)$ and $6 \times 2^{\mathrm{g}}-6$ for the pairs $(4,5)$ and $(5,6)$.

3.1.1. Theorem: $\mathrm{SK}_{\mathrm{N}}(\mathrm{PETIM})=104 \times 2^{\mathrm{g}}-108$.

Proof:

$$
\begin{aligned}
& \mathrm{SK}_{\mathrm{N}}(\text { PETIM })=\sum_{u v \in E} \frac{S(u)+S(v)}{2} \\
& =2 \times 2^{\mathrm{g}}\left(\frac{2+3}{2}\right)+2 \times 2^{\mathrm{g}}\left(\frac{3+4}{2}\right)+\left(8 \times 2^{\mathrm{g}}-12\right)\left(\frac{4+4}{2}\right)+\left(6 \times 2^{\mathrm{g}}-6\right)\left(\frac{4+5}{2}\right)+\left(6 \times 2^{\mathrm{g}}-6\right)\left(\frac{5+6}{2}\right) \\
& =\left(5 \times 2^{\mathrm{g}}\right)+\left(7 \times 2^{\mathrm{g}}\right)+4\left(8 \times 2^{\mathrm{g}}-12\right)+(9 \times 3)\left(2^{\mathrm{g}}-1\right)+(11 \times 3)\left(2^{\mathrm{g}}-1\right)
\end{aligned}
$$


$=104 \times 2^{\mathrm{g}}-108$.

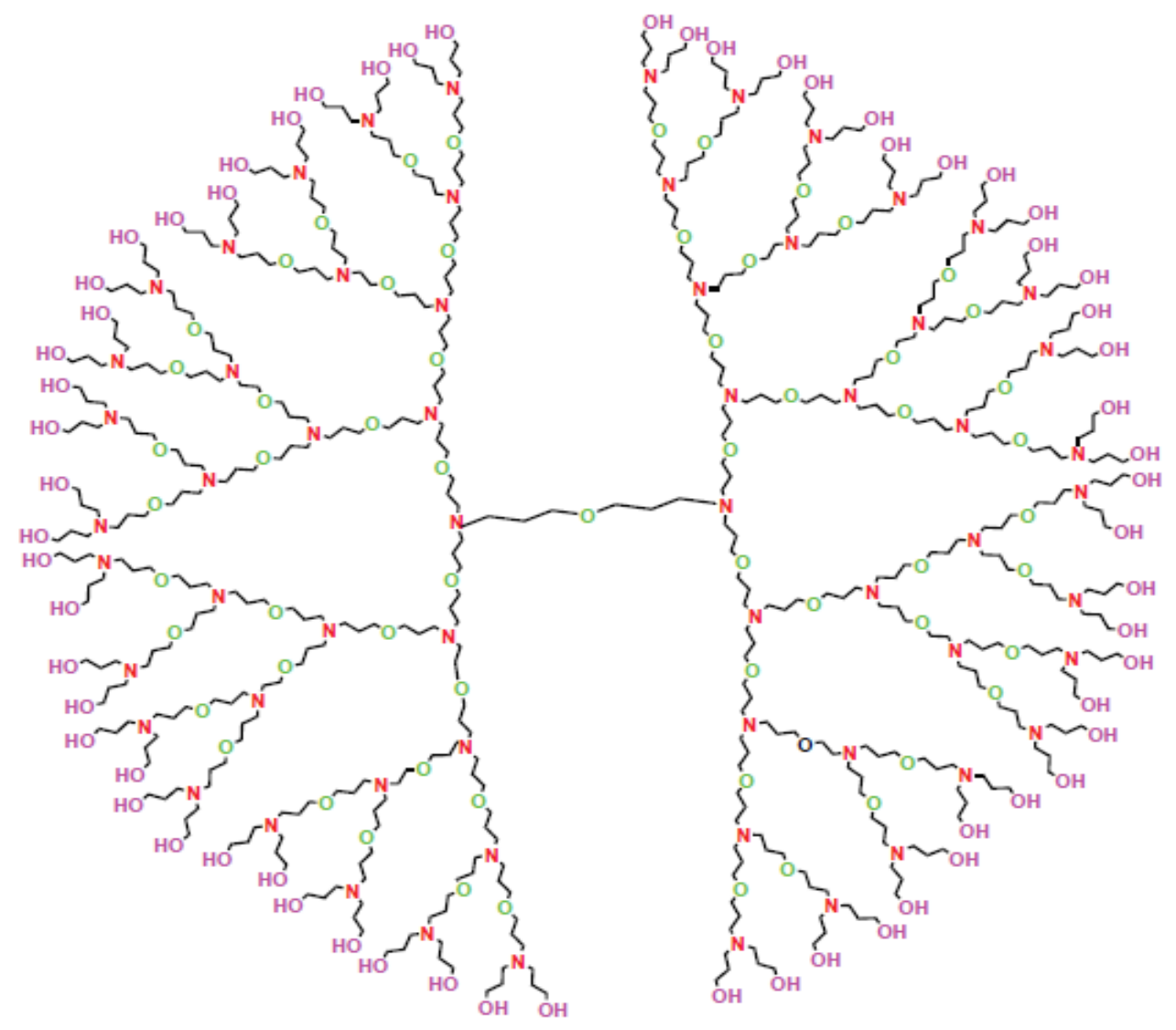

Figure 1. PETIM dendrimer molecular structure.

3.1.2. Theorem SK1 $1_{\mathrm{N}}(\mathrm{PETIM})=232 \times 2^{\mathrm{g}}-246$.

Proof:

$\mathrm{SK} 1_{\mathrm{N}}(\mathrm{PETIM})=\sum_{u v \in E} \frac{S(u) \times S(v)}{2}$

$=2 \times 2^{\mathrm{g}}\left(\frac{2 \times 3}{2}\right)+2 \times 2^{\mathrm{g}}\left(\frac{3 \times 4}{2}\right)+\left(8 \times 2^{\mathrm{g}}-12\right)\left(\frac{4 \times 4}{2}\right)+\left(6 \times 2^{\mathrm{g}}-6\right)\left(\frac{4 \times 5}{2}\right)$

$+\left(6 \times 2^{\mathrm{g}}-6\right)\left(\frac{5 \times 6}{2}\right)$

$=\left(2 \times 2^{\mathrm{g}} \times 3\right)+\left(2 \times 2^{\mathrm{g}} \times 6\right)+8\left(8 \times 2^{\mathrm{g}}-12\right)+10\left(6 \times 2^{\mathrm{g}}-6\right)+15\left(6 \times 2^{\mathrm{g}}-6\right)$

$=232 \times 2^{\mathrm{g}}-246$.

3.1.3. Theorem SK2 ${ }_{\mathrm{N}}($ PETIM $)=468 \times 2^{\mathrm{g}}-495$.

Proof:

SK2N $($ PETIM $)=\sum_{u v \in E}\left(\frac{S(u)+S(v)}{2}\right)^{2}$

$=2 \times 2^{\mathrm{g}}\left(\frac{2+3}{2}\right)^{2}+2 \times 2^{\mathrm{g}}\left(\frac{3+4}{2}\right)^{2}+\left(8 \times 2^{\mathrm{g}}-12\right)\left(\frac{4+4}{2}\right)^{2}+\left(6 \times 2^{\mathrm{g}}-6\right)\left(\frac{4+5}{2}\right)^{2}$

$+\left(6 \times 2^{\mathrm{g}}-6\right)\left(\frac{5+6}{2}\right)^{2}$

$=\left(\frac{25}{2} \times 2^{\mathrm{g}}\right)+\left(\frac{49}{2} \times 2^{\mathrm{g}}\right)+\frac{64}{2}\left(4 \times 2^{\mathrm{g}}-6\right)+\frac{81}{2}\left(3 \times 2^{\mathrm{g}}-3\right)+\frac{121}{2}\left(3 \times 2^{\mathrm{g}}-3\right)$

$=468 \times 2^{\mathrm{g}}-495$.

3.1.4. Theorem: $\mathrm{mR}_{\mathrm{N}}(\mathrm{PETIM})=\frac{161}{30} \times 2^{\mathrm{g}}-\frac{26}{5}$. 
Proof:

$\operatorname{mR}_{\mathrm{N}}(\mathrm{PETIM})=\sum_{u v \in E}\left(\frac{1}{\max \{S(u), S(v)\}}\right)$

$=\left(2 \times 2^{\mathrm{g}}\right)\left(\frac{1}{\max \{2,3\}}\right)+\left(2 \times 2^{\mathrm{g}}\right)\left(\frac{1}{\max \{3,4\}}\right)+\left(8 \times 2^{\mathrm{g}}-12\right)\left(\frac{1}{\max \{4,4\}}\right)$

$+\left(6 \times 2^{\mathrm{g}}-6\right)\left(\frac{1}{\max \{4,5\}}\right)+\left(6 \times 2^{\mathrm{g}}-6\right)\left(\frac{1}{\max \{5,6\}}\right)$

$=\left(2 \times 2^{\mathrm{g}} \times \frac{1}{3}\right)+\left(2 \times 2^{\mathrm{g}} \times \frac{1}{4}\right)+\frac{1}{4}\left(8 \times 2^{\mathrm{g}}-12\right)+\frac{1}{5}\left(6 \times 2^{\mathrm{g}}-6\right)+\frac{1}{6}\left(6 \times 2^{\mathrm{g}}-6\right)$

$=\frac{161}{30} \times 2^{\mathrm{g}}-\frac{26}{5}$.

3.1.5. Theorem $\operatorname{ISI}_{\mathrm{N}}(\mathrm{PETIM})=\frac{59512}{1155} \times 2^{\mathrm{g}}-\frac{14176}{264}$.

Proof:

$\operatorname{ISI}_{\mathrm{N}}(\mathrm{PETIM})=\sum_{u v \in E}\left(\frac{S(u) \times S(v)}{S(u)+S(v)}\right)$

$=\left(2 \times 2^{\mathrm{g}}\right)\left(\frac{2 \times 3}{2+3}\right)+\left(2 \times 2^{\mathrm{g}}\right)\left(\frac{3 \times 4}{3+4}\right)+\left(8 \times 2^{\mathrm{g}}-12\right)\left(\frac{4 \times 4}{4+4}\right)+\left(6 \times 2^{\mathrm{g}}-6\right)\left(\frac{4 \times 5}{4+5}\right)$

$+\left(6 \times 2^{\mathrm{g}}-6\right)\left(\frac{5 \times 6}{5+6}\right)$

$=\left(2 \times 2^{\mathrm{g}} \times \frac{6}{5}\right)+\left(2 \times 2^{\mathrm{g}} \times \frac{12}{7}\right)+\frac{16}{8}\left(8 \times 2^{\mathrm{g}}-12\right)+\frac{20}{9}\left(6 \times 2^{\mathrm{g}}-6\right)+\frac{30}{11}\left(6 \times 2^{\mathrm{g}}-6\right)$

$=\frac{59512}{1155} \times 2^{\mathrm{g}}-\frac{14176}{264}$.

\section{2. $D P Z_{g}$ dendrimer.}

$\mathrm{DPZ}_{\mathrm{g}}$ has four identical branches and a central core in its molecular graph. $\mathrm{DPZ}_{\mathrm{g}}$ has $96 \mathrm{~g}-10$ atoms and $105 \mathrm{~g}-11$ bonds in its molecular graph. The edge-set partition of $\mathrm{DPZ}_{\mathrm{g}}$ are shown [11] as $8 \times 2^{\mathrm{g}}$ for the pair $(4,4), 8$ for the pairs $(4,5),(5,8),(8,9)$ and $(8.10), 16 \times 2^{\mathrm{g}_{-}} 12$ for the pairs $(5,5)$ and $(5,7), 8 \times 2^{\mathrm{g}}-12$ for the pair $(5,6), 8 \times 2^{\mathrm{g}}-12$ for the pair $(7,8), 4 \times 2^{\mathrm{g}}$ for the pair $(6,6), 4$ for the pairs $(6,7),(7,9)$ and $(10,12), 4 \times 2^{g}-4$ for the pair $(6,8)$.Figure 2 depicts the chemical structure of the zinc porphyrin dendrimer $\mathrm{DPZ}_{\mathrm{g}}$.

Theorem 3.2.1: $\mathrm{SK}_{\mathrm{N}}(\mathrm{DPZ})=324 \times 2^{\mathrm{g}}+44$.

Proof:

$\mathrm{SK}_{\mathrm{N}}\left(\mathrm{DPZ} Z_{\mathrm{g}}\right)=\left(8 \times 2^{\mathrm{g}}\right)\left(\frac{4+4}{2}\right)+8\left(\frac{4+5}{2}\right)+\left(16 \times 2^{\mathrm{g}}-12\right)\left(\frac{5+5}{2}\right)+\left(8 \times 2^{\mathrm{g}}-12\right)\left(\frac{5+6}{2}\right)$

$+\left(16 \times 2^{\mathrm{g}}-12\right)\left(\frac{5+7}{2}\right)+8\left(\frac{5+8}{2}\right)+\left(4 \times 2^{\mathrm{g}}\right)\left(\frac{6+6}{2}\right)+4\left(\frac{6+7}{2}\right)+\left(4 \times 2^{\mathrm{g}}-4\right)\left(\frac{6+8}{2}\right)$

$+\left(8 \times 2^{\mathrm{g}}-8\right)\left(\frac{7+8}{2}\right)+4\left(\frac{7+9}{2}\right)+8\left(\frac{8+9}{2}\right)+8\left(\frac{8+10}{2}\right)+4\left(\frac{10+12}{2}\right)$

$=\left(32 \times 2^{\mathrm{g}}\right)+36+\left(80 \times 2^{\mathrm{g}}\right)-60+\left(4 \times 2^{\mathrm{g}}\right)-66+\left(96 \times 2^{\mathrm{g}}\right)-72+52+\left(24 \times 2^{\mathrm{g}}\right)+26$

$+\left(28 \times 2^{\mathrm{g}}\right)-28+\left(60 \times 2^{\mathrm{g}}\right)-60+32+68+72+44$

$=324 \times 2^{\mathrm{g}}+44$.

3.2.2. Theorem $\mathrm{SK} 1_{\mathrm{N}}(\mathrm{DPZ})=1056 \times 2^{\mathrm{g}}+438$.

Proof:

$\mathrm{SK} 1_{\mathrm{N}}\left(\mathrm{DPZ} Z_{\mathrm{g}}\right)=\left(8 \times 2^{\mathrm{g}}\right)\left(\frac{4 \times 4}{2}\right)+8\left(\frac{5 \times 4}{2}\right)+\left(16 \times 2^{\mathrm{g}}-12\right)\left(\frac{5 \times 5}{2}\right)+\left(8 \times 2^{\mathrm{g}}-12\right)\left(\frac{5 \times 6}{2}\right)$

$+\left(16 \times 2^{\mathrm{g}}-12\right)\left(\frac{5 \times 7}{2}\right)+8\left(\frac{5 \times 8}{2}\right)+\left(4 \times 2^{\mathrm{g}}\right)\left(\frac{6 \times 6}{2}\right)+4\left(\frac{6 \times 7}{2}\right)+\left(4 \times 2^{\mathrm{g}}-4\right)\left(\frac{6 \times 8}{2}\right)$ 
$+\left(8 \times 2^{\mathrm{g}}-8\right)\left(\frac{7 \times 8}{2}\right)+4\left(\frac{7 \times 9}{2}\right)+8\left(\frac{8 \times 9}{2}\right)+8\left(\frac{8 \times 10}{2}\right)+4\left(\frac{10 \times 12}{2}\right)$

$=\left(8 \times 2^{\mathrm{g}} \times 8\right)+80+\frac{25}{2}\left(16 \times 2^{\mathrm{g}}-12\right)+15\left(8 \times 2^{\mathrm{g}}-12\right)+\frac{35}{2}\left(16 \times 2^{\mathrm{g}}-12\right)+160+(18 \times 4$ $\left.\mathrm{x} 2^{\mathrm{g}}\right)$

$+84+24\left(4 \times 2^{\mathrm{g}}-4\right)+28\left(8 \times 2^{\mathrm{g}}-8\right)+126+288+320+240$

$=1056 \times 2^{\mathrm{g}}+438$.

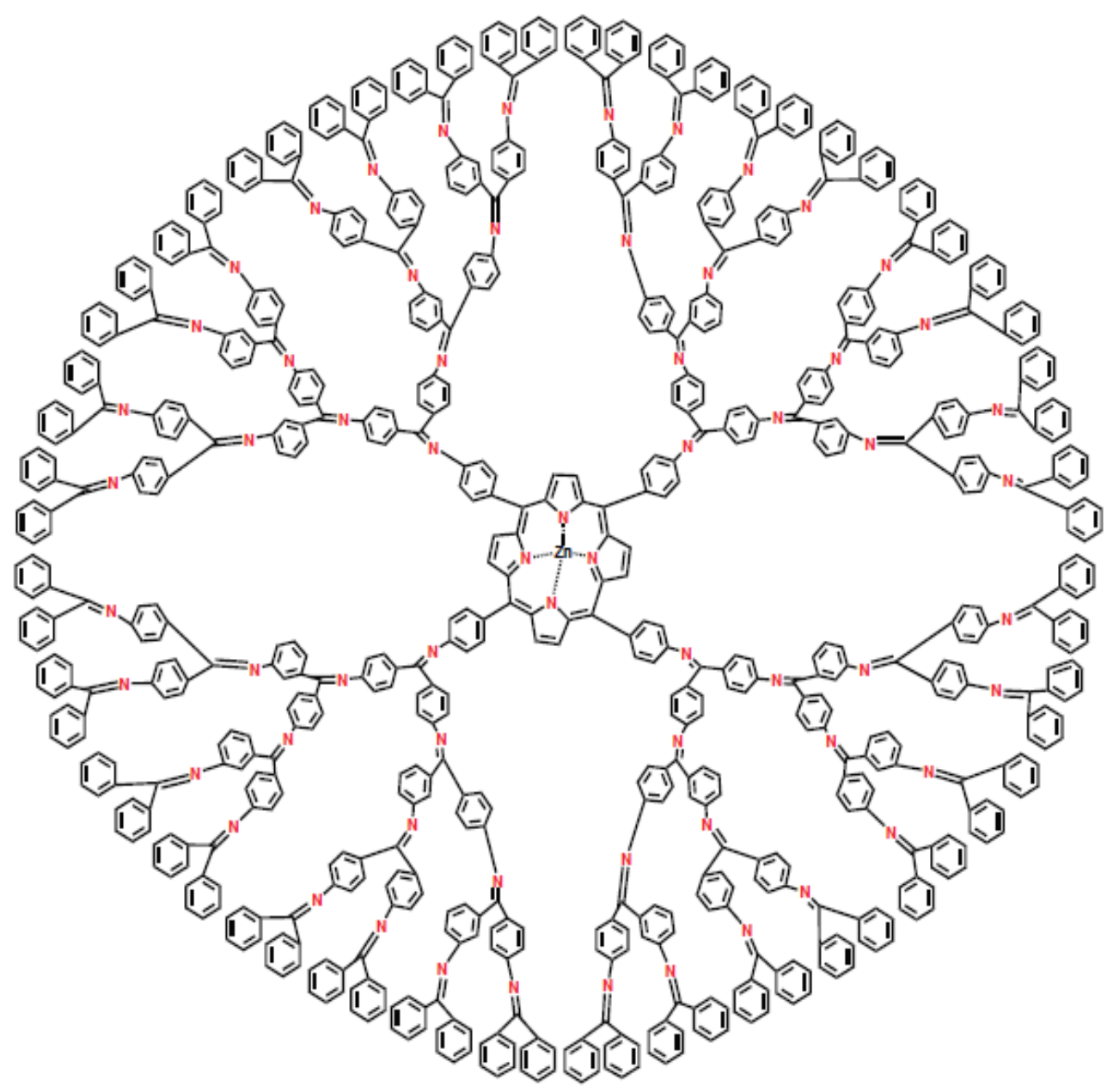

Figure 2. Zinc porphyrin dendrimer $\mathrm{DPZ}_{\mathrm{g}}$ molecular structure.

3.2.3. Theorem SK2 ${ }_{\mathrm{N}}(\mathrm{DPZ})=2136 \times 2^{\mathrm{g}}+894$.

Proof:

$\mathrm{SK} 2 \mathrm{~N}(\mathrm{DPZ})=\left(8 \times 2^{\mathrm{g}}\right)\left(\frac{4+4}{2}\right)^{2}+8\left(\frac{5+4}{2}\right)^{2}+\left(16 \times 2^{\mathrm{g}}-12\right)\left(\frac{5+5}{2}\right)^{2}+\left(8 \times 2^{\mathrm{g}}-12\right)\left(\frac{5+6}{2}\right)^{2}$

$+\left(16 \times 2^{\mathrm{g}}-12\right)\left(\frac{5+7}{2}\right)^{2}+8\left(\frac{5+8}{2}\right)^{2}+\left(4 \times 2^{\mathrm{g}}\right)\left(\frac{6+6}{2}\right)^{2}+4\left(\frac{6+7}{2}\right)^{2}+\left(4 \times 2^{\mathrm{g}}-4\right)\left(\frac{6+8}{2}\right)^{2}$

$+\left(8 \times 2^{\mathrm{g}}-8\right)\left(\frac{7+8}{2}\right)^{2}+4\left(\frac{7+9}{2}\right)^{2}+8\left(\frac{8+9}{2}\right)^{2}+8\left(\frac{8+10}{2}\right)^{2}+4\left(\frac{10+12}{2}\right)^{2}$

$=\left(8 \times 16 \times 2^{\mathrm{g}}\right)+(2 \times 81)+\left(16 \times 25 \times 2^{\mathrm{g}}\right)-(12 \times 25)+\left(121 \times 2 \times 2^{\mathrm{g}}\right)-(121 \times 3)$

$+\left(36 \times 16 \times 2^{\mathrm{g}}\right)-(36 \times 12)+(2 \times 169)+\left(361 \times 4 \times 2^{\mathrm{g}}\right)+169+\left(49 \times 4 \times 2^{\mathrm{g}}\right)$

$-(49 \times 4)+\left(225 \times 2 \times 2^{\mathrm{g}}\right)-(225 \times 2)+(4 \times 64)+(2 \times 289)+(8 \times 81)+484$

$=2136 \times 2^{\mathrm{g}}+894$.

3.2.4. Theorem $m R_{\mathrm{N}}\left(\mathrm{DPZ}_{\mathrm{g}}\right)=\frac{2307}{210} \times 2^{\mathrm{g}}-\frac{41}{42}$.

Proof: 


$$
\begin{aligned}
& \mathrm{mR}_{\mathrm{N}}(\mathrm{DPZ} \mathrm{g})=\left(8 \times 2^{\mathrm{g}}\right)\left(\frac{1}{\max \{4,4\}}\right)+8\left(\frac{1}{\max \{5,4\}}\right)+\left(16 \times 2^{\mathrm{g}}-12\right)\left(\frac{1}{\max \{5,5\}}\right) \\
& +\left(8 \times 2^{\mathrm{g}}-12\right)\left(\frac{1}{\max \{5,6\}}\right)+\left(16 \times 2^{\mathrm{g}}-12\right)\left(\frac{1}{\max \{5,7\}}\right)+8\left(\frac{1}{\max \{5,8\}}\right)+\left(4 \times 2^{\mathrm{g}}\right)\left(\frac{1}{\max \{6,6\}}\right) \\
& +4\left(\frac{1}{\max \{6,7\}}\right)+\left(4 \times 2^{\mathrm{g}}-4\right)\left(\frac{1}{\max \{6,8\}}\right)+\left(8 \times 2^{\mathrm{g}}-8\right)\left(\frac{1}{\max \{7,8\}}\right)+4\left(\frac{1}{\max \{7,9\}}\right) \\
& +8\left(\frac{1}{\max \{8,9\}}\right)+8\left(\frac{1}{\max \{8,10\}}\right)+4\left(\frac{1}{\max \{10,12\}}\right) \\
& =\frac{1}{4}\left(8 \times 2^{\mathrm{g}}\right)+8\left(\frac{1}{5}\right)+\frac{1}{5}\left(16 \times 2^{\mathrm{g}}-12\right)+\frac{1}{6}\left(8 \times 2^{\mathrm{g}}-12\right)+\frac{1}{7}\left(16 \times 2^{\mathrm{g}}-12\right)+8\left(\frac{1}{8}\right) \\
& +\frac{1}{6}\left(4 \times 2^{\mathrm{g}}\right)+4\left(\frac{1}{7}\right)+\frac{1}{8}\left(4 \times 2^{\mathrm{g}}-4\right)+\frac{1}{8}\left(8 \times 2^{\mathrm{g}}-8\right)+4\left(\frac{1}{9}\right)+8\left(\frac{1}{9}\right)+8\left(\frac{1}{10}\right)+4\left(\frac{1}{12}\right) \\
& =\frac{2307}{210} \times 2^{\mathrm{g}}-\frac{41}{42} .
\end{aligned}
$$

3.2.5. Theorem $\operatorname{ISI}_{\mathrm{N}}(\mathrm{DPZ})=\frac{215522}{1155} \times 2^{\mathrm{g}}-\frac{64367463}{3063060}$.

Proof:

$$
\begin{aligned}
& \operatorname{ISI}_{N}\left(D P Z_{g}\right)=\left(8 \times 2^{\mathrm{g}}\right)\left(\frac{4 \times 4}{4+4}\right)+8\left(\frac{5 \times 4}{5+4}\right)+\left(16 \times 2^{\mathrm{g}}-12\right)\left(\frac{5 \times 5}{5+5}\right)+\left(8 \times 2^{\mathrm{g}}-12\right)\left(\frac{5 \times 6}{5+6}\right) \\
& +\left(16 \times 2^{\mathrm{g}}-12\right)\left(\frac{5 \times 7}{5+7}\right)+8\left(\frac{5 \times 8}{5+8}\right)+\left(4 \times 2^{\mathrm{g}}\right)\left(\frac{6 \times 6}{6+6}\right)+4\left(\frac{6 \times 7}{6+7}\right)+\left(4 \times 2^{\mathrm{g}}-4\right)\left(\frac{6 \times 8}{6+8}\right) \\
& +\left(8 \times 2^{\mathrm{g}}-8\right)\left(\frac{7 \times 8}{7+8}\right)+4\left(\frac{7 \times 9}{7+9}\right)+8\left(\frac{8 \times 9}{8+9}\right)+8\left(\frac{8 \times 10}{8+10}\right)+4\left(\frac{10 \times 12}{10+12}\right) \\
& =\frac{215522}{1155} \times 2^{\mathrm{g}}-\frac{64367463}{3063060} .
\end{aligned}
$$

\section{3. $D_{g} P_{g}$ dendrimer.}

$\mathrm{D}_{\mathrm{g}} \mathrm{P}_{\mathrm{g}}$ molecular graph comprises four comparable wings and a center core with five more edges (Figures 3 and 4). The total number of atoms in porphyrin dendrimer [11] is $96 \mathrm{~g}-$ 10 , and the number of bonds in $\mathrm{D}_{\mathrm{g}} \mathrm{P}_{\mathrm{g}}$ is $105 \mathrm{~g}-11$. Note that $\mathrm{g}=2^{\mathrm{t}}$, wheret $\geq 2$. The edge-set partition of $\mathrm{D}_{\mathrm{g}} \mathrm{P}_{\mathrm{g}}$ are given as $2 \mathrm{~g}, \mathrm{~g}+1,8 \mathrm{~g}-6,24 \mathrm{~g}, 4 \mathrm{~g}, 4 \mathrm{~g}-6,6 \mathrm{~g}, 18 \mathrm{~g}, 25 \mathrm{~g}, 11 \mathrm{~g}$ for the pairs $(3,5)$, $(4,4),(4,5),(4,6),(5,5),(5,6),(5,7),(6,7),(6,8)$ and $(7,9)$ respectively and $g$ for the pairs $(7,8)$ and $(8,9)$.

\subsubsection{Theorem $\mathrm{SK}_{\mathrm{N}}\left(\mathrm{D}_{\mathrm{g}} \mathrm{P}_{\mathrm{g}}\right)=642 \mathrm{~g}-56$.}

Proof:

$\mathrm{SK}_{\mathrm{N}}\left(\mathrm{D}_{\mathrm{g}} \mathrm{P}_{\mathrm{g}}\right)=2 \mathrm{~g}\left(\frac{3+5}{2}\right)+(\mathrm{g}+1)\left(\frac{4+4}{2}\right)+(8 \mathrm{~g}-6)\left(\frac{4+5}{2}\right)+24 \mathrm{~g}\left(\frac{4+6}{2}\right)+4 \mathrm{~g}\left(\frac{5+5}{2}\right)$

$+(4 \mathrm{~g}-6)\left(\frac{5+6}{2}\right)+6 \mathrm{~g}\left(\frac{5+7}{2}\right)+18 \mathrm{~g}\left(\frac{6+7}{2}\right)+25 \mathrm{~g}\left(\frac{6+8}{2}\right)+\mathrm{g}\left(\frac{7+8}{2}\right)+11 \mathrm{~g}\left(\frac{7+9}{2}\right)+\mathrm{g}\left(\frac{8+9}{2}\right)$

$=8 \mathrm{~g}+4 \mathrm{~g}+4+36 \mathrm{~g}-27+120 \mathrm{~g}+20 \mathrm{~g}+22 \mathrm{~g}-33+36 \mathrm{~g}+117 \mathrm{~g}+175 \mathrm{~g}$

$+\frac{15 g}{2}+88 \mathrm{~g}+\frac{17 g}{2}$

$=642 \mathrm{~g}-56$.

\subsubsection{Theorem $\mathrm{SK} 1_{\mathrm{N}}\left(\mathrm{D}_{\mathrm{g}} \mathrm{P}_{\mathrm{g}}\right)=1994.5 \mathrm{~g}-142$.}

Proof:

$$
\begin{aligned}
& \text { SK } 1_{N}\left(D_{g} P_{g}\right)=2 g\left(\frac{3 \times 5}{2}\right)+(g+1)\left(\frac{4 \times 4}{2}\right)+(8 g-6)\left(\frac{4 \times 5}{2}\right)+24 g\left(\frac{4 \times 6}{2}\right)+4 g\left(\frac{5 \times 5}{2}\right) \\
& +(4 g-6)\left(\frac{5 \times 6}{2}\right)+6 g\left(\frac{5 \times 7}{2}\right)+18 g\left(\frac{6 \times 7}{2}\right)+25 g\left(\frac{6 \times 8}{2}\right)+g\left(\frac{7 \times 8}{2}\right)+11 g\left(\frac{7 \times 9}{2}\right)+g\left(\frac{8 \times 9}{2}\right) \\
& =15 g+8 g+8+80 g-60+288 g+50 g+60 g-90+105 g+378 g+600 g
\end{aligned}
$$




$$
\begin{aligned}
& +28 g+\frac{693 g}{2}+36 g \\
& =1994.5 \mathrm{~g}-142 .
\end{aligned}
$$

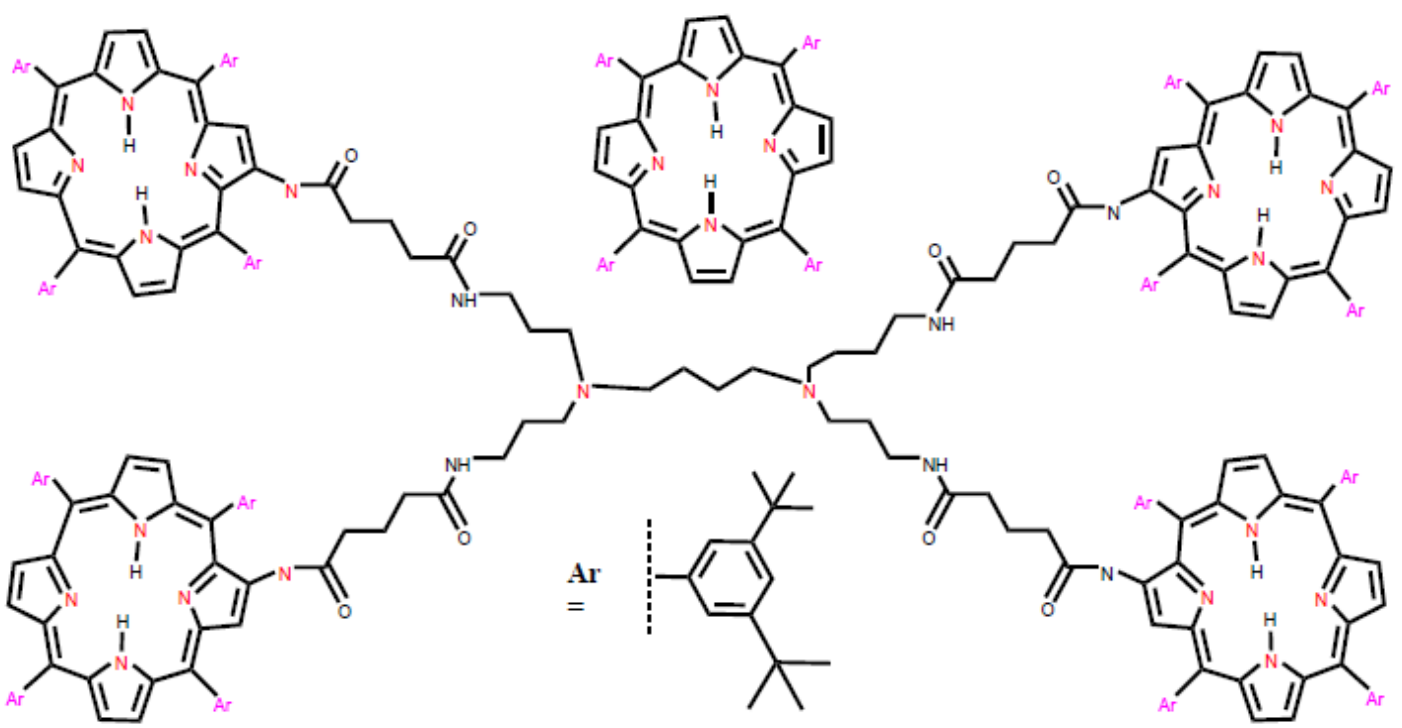

Figure 3. Porphyrin DendrimerD $\mathrm{D}_{4}$ molecular graph.

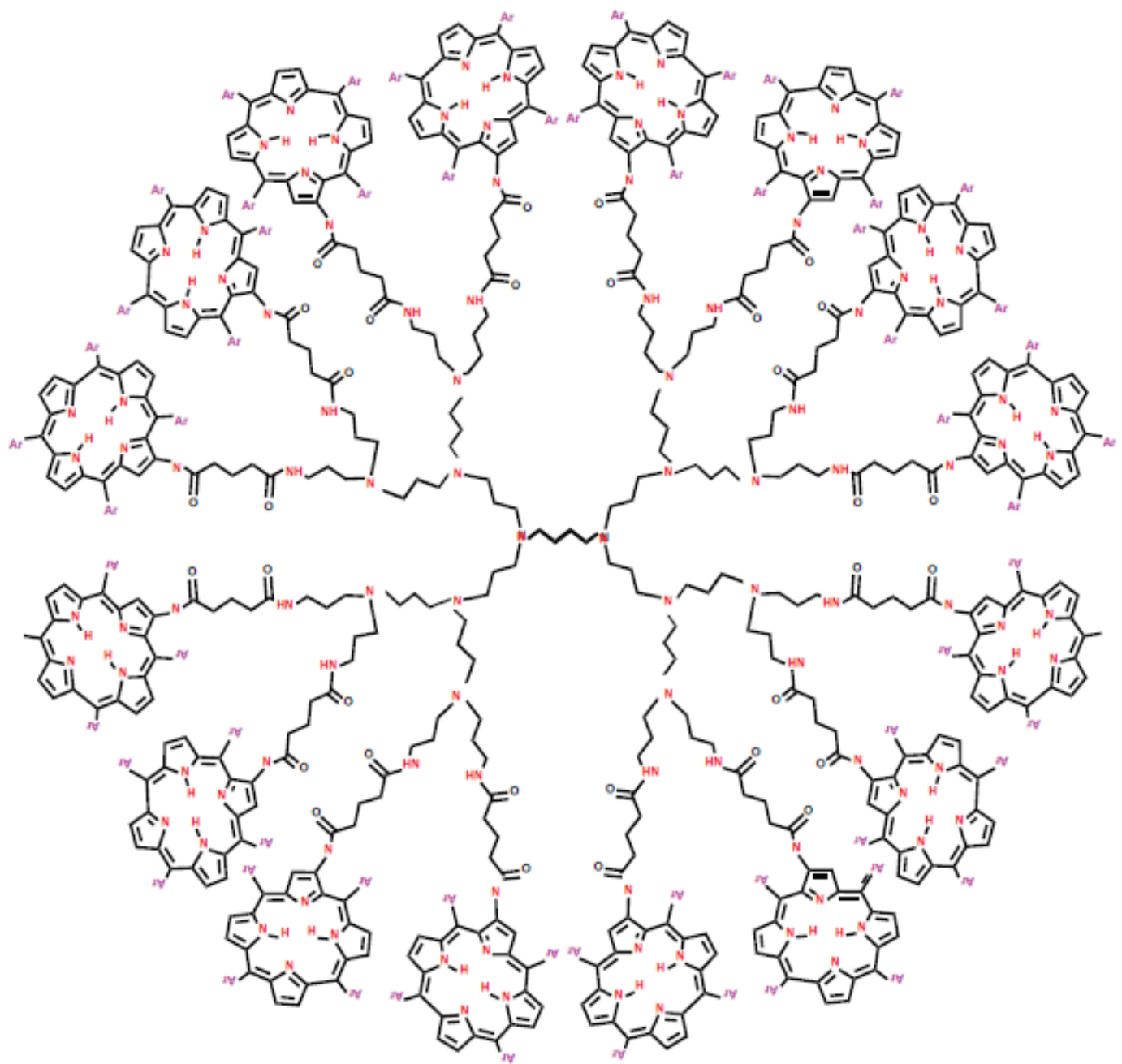

Figure 4. Porphyrin Dendrimer $\mathrm{D}_{16} \mathrm{P}_{16}$ molecular graph. 
3.3.3. Theorem $\mathrm{SK} 2_{\mathrm{N}}\left(\mathrm{D}_{\mathrm{g}} \mathrm{P}_{\mathrm{g}}\right)$ is $4065 \mathrm{~g}-287$.

Proof:

$\mathrm{SK} 2 \mathrm{~N}\left(\mathrm{D}_{\mathrm{g}} \mathrm{P}_{\mathrm{g}}\right)=2 \mathrm{~g}\left(\frac{3+5}{2}\right)^{2}+(\mathrm{g}+1)\left(\frac{4+4}{2}\right)^{2}+(8 \mathrm{~g}-6)\left(\frac{4+5}{2}\right)^{2}+24 \mathrm{~g}\left(\frac{4+6}{2}\right)^{2}+4 \mathrm{~g}\left(\frac{5+5}{2}\right)^{2}$

$+(4 \mathrm{~g}-6)\left(\frac{5+6}{2}\right)^{2}+6 \mathrm{~g}\left(\frac{5+7}{2}\right)^{2}+18 \mathrm{~g}\left(\frac{6+7}{2}\right)^{2}+25 \mathrm{~g}\left(\frac{6+8}{2}\right)^{2}+\mathrm{g}\left(\frac{7+8}{2}\right)^{2}+11 \mathrm{~g}\left(\frac{7+9}{2}\right)^{2}+\mathrm{g}\left(\frac{8+9}{2}\right)^{2}$

$=4065 \mathrm{~g}-287$.

3.3.4. Theorem $m R_{N}\left(D_{g} P_{g}\right)=\frac{1101 g}{70}-\frac{39}{20}$.

Proof:

$\mathrm{mR}_{\mathrm{N}}\left(\mathrm{D}_{\mathrm{g}} \mathrm{P}_{\mathrm{g}}\right)=2 \mathrm{~g}\left(\frac{1}{\max \{3,5\}}\right)+(\mathrm{g}+1)\left(\frac{1}{\max \{4,4\}}\right)+(8 \mathrm{~g}-6)\left(\frac{1}{\max \{4,5\}}\right)+24 \mathrm{~g}\left(\frac{1}{\max \{4,6\}}\right)$

$+4 \mathrm{~g}\left(\frac{1}{\max \{5,5\}}\right)+(4 \mathrm{~g}-6)\left(\frac{1}{\max \{5,6\}}\right)+6 \mathrm{~g}\left(\frac{1}{\max \{5,7\}}\right)+18 \mathrm{~g}\left(\frac{1}{\max \{6,7\}}\right)$

$+25 \mathrm{~g}\left(\frac{1}{\max \{6,8\}}\right)+\mathrm{g}\left(\frac{1}{\max \{7,8\}}\right)+11 \mathrm{~g}\left(\frac{1}{\max \{7,9\}}\right)+\mathrm{g}\left(\frac{1}{\max \{8,9\}}\right)$

$=2 \mathrm{~g}\left(\frac{1}{5}\right)+(\mathrm{g}+1)\left(\frac{1}{4}\right)+(8 \mathrm{~g}-6)\left(\frac{1}{5}\right)+24 g\left(\frac{1}{6}\right)+4 \mathrm{~g}\left(\frac{1}{5}\right)+(4 \mathrm{~g}-6)\left(\frac{1}{6}\right)+6 \mathrm{~g}\left(\frac{1}{7}\right)$

$+18 \mathrm{~g}\left(\frac{1}{7}\right)+25 \mathrm{~g}\left(\frac{1}{8}\right)+\mathrm{g}\left(\frac{1}{8}\right)+11 \mathrm{~g}\left(\frac{1}{9}\right)+g\left(\frac{1}{9}\right)$

$=\frac{1101 g}{70}-\frac{39}{20}$.

3.3.5. Theorem $\operatorname{ISI}_{\mathrm{N}}\left(\mathrm{D}_{\mathrm{g}} \mathrm{P}_{\mathrm{g}}\right)=\frac{771121993 g}{2450448}-\frac{914}{33}$.

Proof:

$\operatorname{ISI}_{\mathrm{N}}\left(\mathrm{D}_{\mathrm{g}} \mathrm{P}_{\mathrm{g}}\right)=2 \mathrm{~g}\left(\frac{3 \times 5}{3+5}\right)+(\mathrm{g}+1)\left(\frac{4 \times 4}{4+4}\right)+(8 \mathrm{~g}-6)\left(\frac{4 \times 5}{4+5}\right)+24 \mathrm{~g}\left(\frac{4 \times 6}{4+6}\right)+4 \mathrm{~g}\left(\frac{5 \times 5}{5+5}\right)+(4 \mathrm{~g}-6)$

$\left(\frac{5 \times 6}{5+6}\right)+6 \mathrm{~g}\left(\frac{5 \times 7}{5+7}\right)+18 \mathrm{~g}\left(\frac{6 \times 7}{6+7}\right)+25 \mathrm{~g}\left(\frac{6 \times 8}{6+8}\right)+\mathrm{g}\left(\frac{7 \times 8}{7+8}\right)+11 \mathrm{~g}\left(\frac{7 \times 9}{7+9}\right)+\mathrm{g}\left(\frac{8 \times 9}{8+9}\right)$

$=\frac{771121993 g}{2450448}-\frac{914}{33}$.

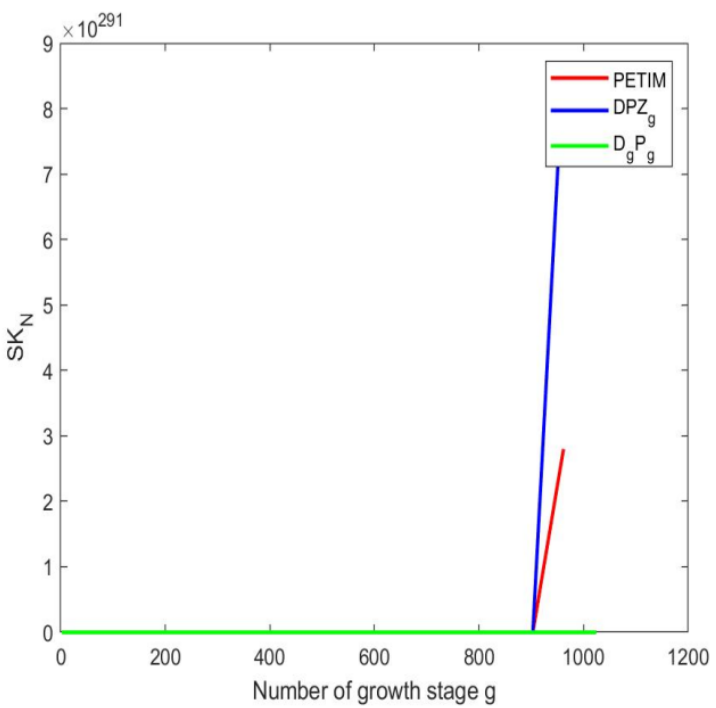

Figure 5. $\mathrm{SK}_{\mathrm{N}}$ index of PETIM, $\mathrm{DPZ}$, and $\mathrm{D}_{\mathrm{g}} \mathrm{P}_{\mathrm{g}}$ dendrimers.

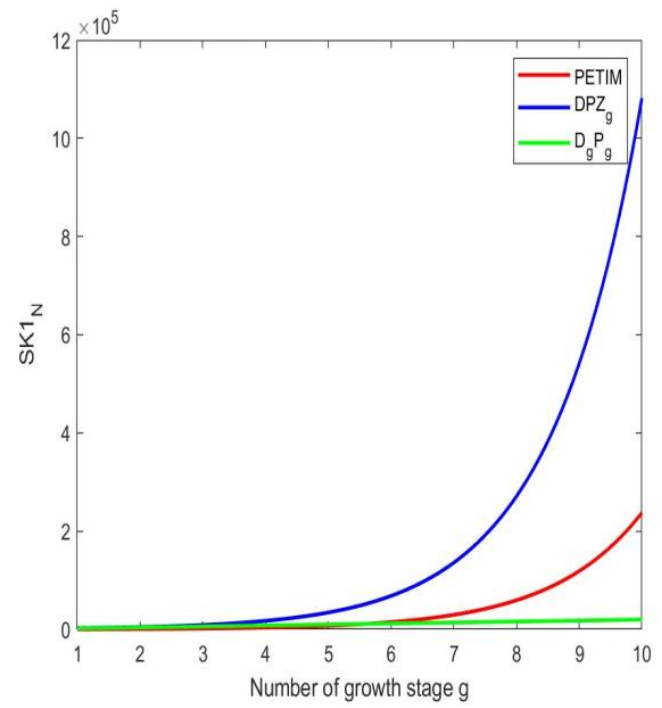

Figure 6. $\mathrm{SK} 1_{\mathrm{N}}$ index of PETIM, $\mathrm{DPZ}$, and $\mathrm{D}_{\mathrm{g}} \mathrm{P}_{\mathrm{g}}$. 

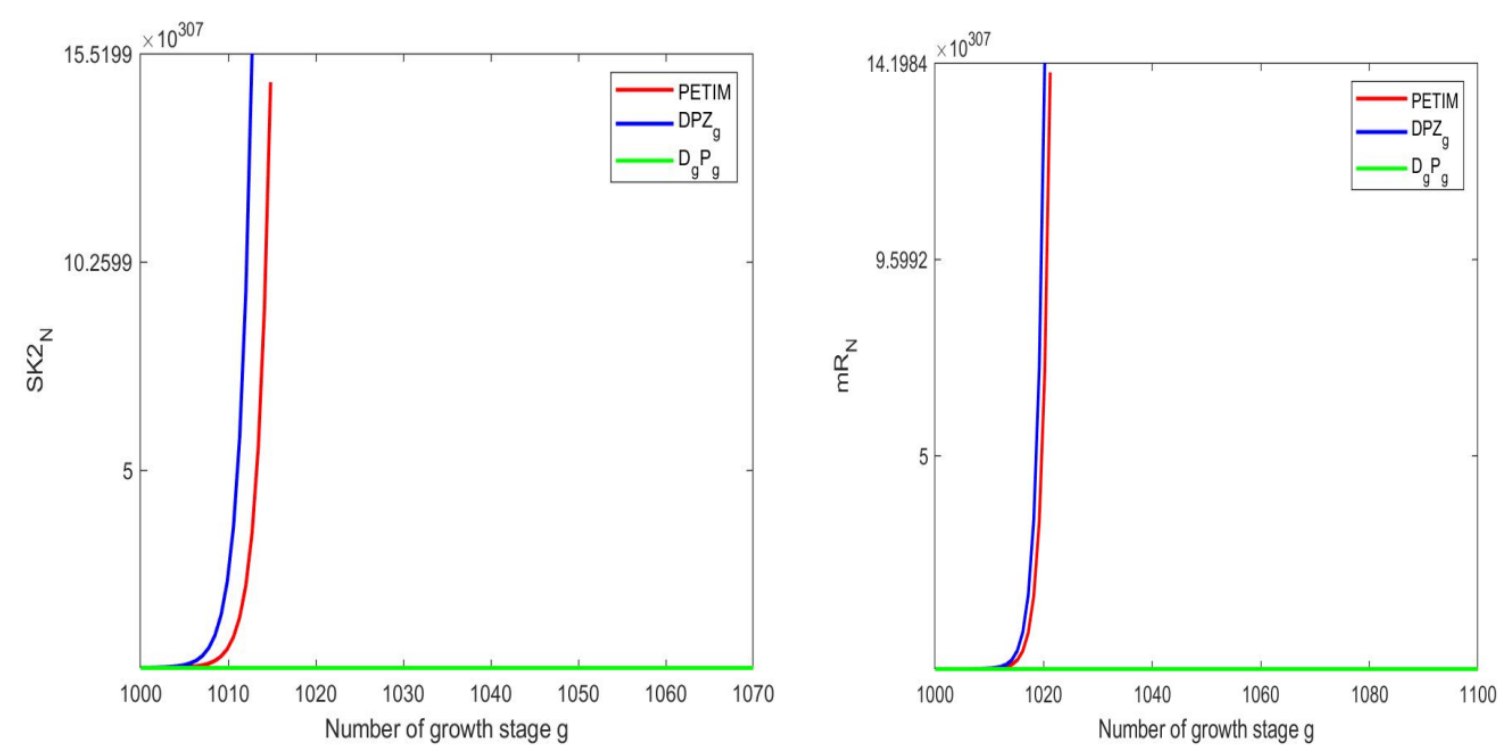

Figure 7. $\mathrm{SK} 2_{\mathrm{N}}$ index of PETIM, $\mathrm{DPZ}$, and $\mathrm{D}_{\mathrm{g}} \mathrm{P}_{\mathrm{g}}$ dendrimers. Figure 8. $\mathrm{mR}_{\mathrm{N}}$ index of PETIM, DPZ $\mathrm{Z}_{\mathrm{g}}$, and $\mathrm{D}_{\mathrm{g}} \mathrm{P}_{\mathrm{g}}$

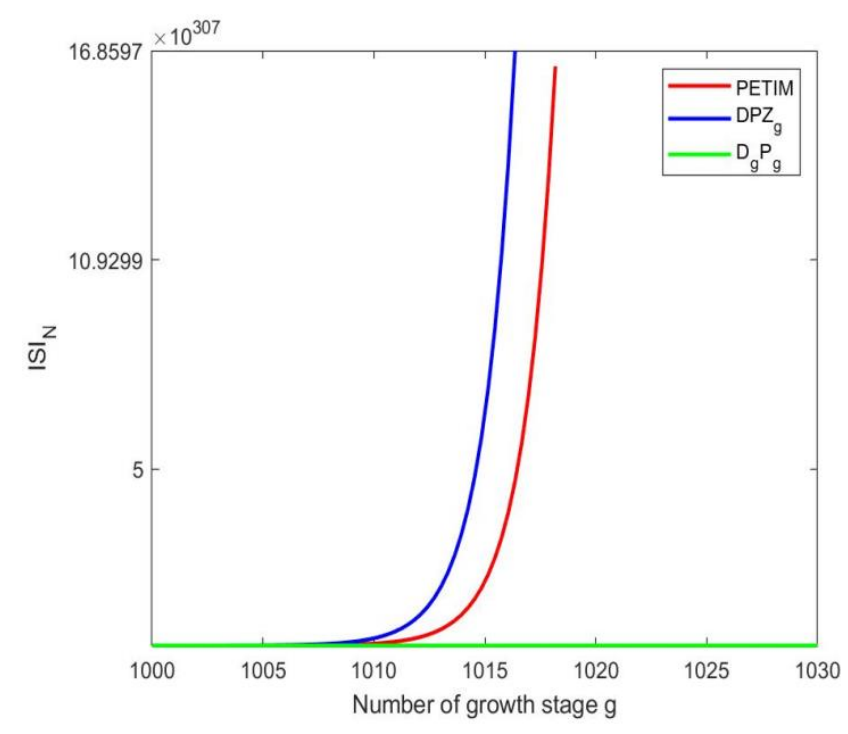

Figure 9. $\mathrm{ISI}_{\mathrm{N}}$ index of PETIM, DPZ $\mathrm{g}$, and $\mathrm{D}_{\mathrm{g}} \mathrm{P}_{\mathrm{g}}$ dendrimers.

\subsection{Graphical representation.}

The graphical representation of $\mathrm{SK}_{\mathrm{N}}, \mathrm{SK} 1_{\mathrm{N}}, \mathrm{SK} 2_{\mathrm{N}}, \mathrm{mR}_{\mathrm{N}}$, and $\mathrm{ISI}_{\mathrm{N}}$ for the dendrimers PETIM, $\mathrm{DPZ}_{\mathrm{g}}$, and $\mathrm{D}_{\mathrm{g}} \mathrm{P}_{\mathrm{g}}$ are given in Figure 5-9. In the following figures, the $\mathrm{x}$-axis represents the number of growth-stage $\mathrm{g}$, and the $\mathrm{y}$-axis represents the corresponding index. PETIM, $\mathrm{DPZ}$, and $\mathrm{D}_{\mathrm{g}} \mathrm{P}_{\mathrm{g}}$ dendrimers are plotted by red, blue, and green colors, respectively. It is observed from the figures that $\mathrm{DPZ}_{\mathrm{g}}$ has a higher value for Neighborhood-based indices than PETIM and $\mathrm{D}_{\mathrm{g}} \mathrm{P}_{\mathrm{g}}$.

\section{Conclusions}

The molecular study and the research of relation between indices and molecular attributes are made possible by computing numerous topological indices of graphs connected with chemical graphs. In this study, we proposed several Neighborhood-based topological indices for dendrimers, namely PETIM, DPZg, and $\mathrm{D}_{\mathrm{g}} \mathrm{P}_{\mathrm{g}}$. Chemistry, physics, and other practical disciplines can benefit from our findings. Topological indices have been shown to aid in predicting various properties without the need for a wet lab. MATLAB was also used to 
illustrate the 2D representations of these indices. We plan to establish the link between these descriptors and various chemical features of dendrimers in the future.

\section{Funding}

This research received no external funding.

\section{Acknowledgments}

This research has no acknowledgment.

\section{Conflicts of Interest}

The authors declare no conflict of interest.

\section{References}

1. Wiener, H. Structural determination of paraffin boiling points. Journal of the American Chemical Society 1947, 69, 17-20,https://doi.org/10.1021/ja01193a005.

2. Randic, M. Characterization of molecular branching. Journal of the American Chemical Society 1975, 97, 6609-6615,https://doi.org/10.1021/ja00856a001.

3. Gutman, I. Molecular graphs with minimal and maximal Randic indices. Croatica Chemica Acta 2002, 75, 357-369, https://hrcak.srce.hr/127525.

4. West, D.B. An Introduction to Graph Theory. 2nded.; Prentice-Hall: New Delhi, India 2002.

5. Subheet Jain; Amanpreet Kaur; Richa Puri; Puneet Utreja; Anubhuti Jain; Mahesh Bhide; Rakesh Ratnam; Vinay Singh; Patil, A.S.; Jayaraman, N.; Gaurav Kaushik; Subodh Yadav; Khanduja, K.L. Poly propyl ether imine (PETIM) dendrimer: A novel non-toxic dendrimer for sustained drug delivery. European Journal of Medicinal Chemistry 2010, 45, 4997-5005, https://doi.org/10.1016/j.ejmech.2010.08.006.

6. Lakshminarayanan, A.; Ravi, V.K.; Tatineni, R.; Rajesh, Y. B. R. D.; Maingi, V.; Vasu, K.S.; Madhusudhan, N.; Maiti, P.K.; Sood, A.K.; Das, S.; Jayaraman, N. Efficient Dendrimer-DNA Complexation and Gene Delivery Vector Properties of Nitrogen-Core Poly(propyl ether imine) Dendrimer in Mammalian Cells.Bioconjugate Chemistry 2013, 24, 1612-1623, https://doi.org/10.1021/bc400247w.

7. Chandan J.; Jayamurugan, G. Structure of poly(propyl ether imine) dendrimer from fully atomistic molecular dynamics simulation and by small angle x-ray scattering.Journal of Chemical Physics 2006, 124, 204719, https://doi.org/10.1063/1.2194538.

8. Basavaraj K. N.; Hiren M. B.; Ganesh K. D.; Manvi, F.V.; Veerendra K. N. Dendrimers: Emerging polymers for drug-delivery systems.European Journal of Pharmaceutical Sciences 2009, 38, 185196,https://doi.org/10.1016/j.ejps.2009.07.008.

9. Astruc, D.; Boisselier, E.; Ornelas, C. Dendrimersdesigned for functions: from physical photophysical and supramolecular properties to applications in sensing catalysis molecular electronics photonics and nanomedicine.Chemical Reviews 2010, 110, 1857-1959, http://doi.org./10.1021/cr900327d.

10. Humberto G.-D.; Santiago V.; Lourdes S.; Eugenio U. Medicinal Chemistry and Bioinformatics - Current Trends in Drugs Discovery with Networks Topological Indices.Current Topics in Medicinal Chemistry 2007, 7, 1015-1029, https://doi.org/10.2174/156802607780906771.

11. Yasir B.; Adnan A.; Muhammad K.; Muhammad I. Q.; Adnan J.; Muhammad R.; Nargis B.; Nazeer M.On Forgotten Topological Indices of Some Dendrimers Structure. Molecules 2017, 22, 867, https://doi.org/10.3390/molecules22060867.

12. Alyar, S.; Khoeilar, R.; Jahanbani, A. Some topological indices of dendrimers. International Journal of Computational Materials Science and Engineering 2020, 9, 2050018, http://doi.org/10.1142/S2047684120500189.

13. Kang, S.M.; Zahid, M.A.; Virk,A.R.; Nazeer, W.; Gao, W. Calculating the Degree-based Topological Indices of Dendrimers.Open Chemistry 2018, 16, 681-688, https://doi.org/10.1515/chem-2018-0071.

14. Gao, W.; Aamir, M.; Iqbal, Z.; Ishaq, M.; Aslam, A. On Irregularity Measures of Some Dendrimers Structures. Mathematics 2019, 7, 271, https://doi.org/10.3390/math7030271.

15. Aslam, A.; Jamil, M.K.; Gao, W.; Nazeer, W. Topological aspects of some dendrimer structures.Nanotechnology Reviews 2018, 7, 123-129, https://doi.org/10.1515/ntrev-2017-0184.

16. Shahid A.; Abaid R.V.; Rehman, M.A.; Nehad A.S. Analysis of Dendrimer Generation by Sombor Indices.Journal of Chemistry 2021, 2021,11, https://doi.org/10.1155/2021/9930645.

17. Aslam, A.; Bashir, Y.; Rafiq, M.; Haider, F.; Muhammad, N.; Bibi, N. Three New/Old Vertex-Degree Based Topological Indices of some Dendrimers Structure. Electronic Journal of Biology 2017, 13. 
18. Diudea, M.; Vizitiu, A.; Mirzagar, M.; Ashrafi, A. Sadhana polynomial in nano-dendrimers. Carpathian Journal of Mathematics 2010, 26, 59-66, https://www.jstor.org/stable/43999432.

19. Zhihua C.; Abaid R.V.; Mustafa H.; Tariq J.Z.; Imran A.; Ce S.; Waqas N. Irregularity Indices of Dendrimer Structures Used as Molecular Disrupter in QSAR Study. Journal of Chemistry 2019, 2019, 21, https://doi.org/10.1155/2019/5371254.

20. Chu, Z.-Q.; Salman, M.; Munir, A.; Khalid, I.; Rehman, M.U.; Liu, J.-B.; Ali, F. Some topological indices of dendrimers determined by their Banhatti polynomials. Heterocyclic Communications 2020, 26, 99-111, https://doi.org/10.1515/hc-2020-0102.

21. Murat C.; Süleyman E.; Hafiz M.-U.-R.; Deeba A. M-polynomial and topological indices Poly (EthyleneAmidoAmine) dendrimers. Journal of Information and Optimization Sciences 2020, 41, 11171131, http://doi.org./10.1080/02522667.2020.1745383.

22. Modjtaba G.; Mina R.-P.; Ottorino O. Distance complexity measures versus the orbit-entropy measure of dendrimers. Fullerenes, Nanotubes and Carbon Nanostructures 2021, https://doi.org/10.1080/1536383X.2021.1955246.

23. Mondal, S.; De, N.; Pal, A. Neighborhood degree sum-based molecular descriptors of fractal and Cayley tree dendrimers. European Physical Journal Plus 2021, 136, 303, https://doi.org/10.1140/epjp/s13360-02101292-4.

24. Mathad, V.; Padmapriya, P.; Sangamesha, M.A.Some topological indices of phosphorus containing dendrimers. Palestine Journal of Mathematics 2021, 10, 151-160.

25. Muhammad K.H.; Faryal C.; Abdul J.M.; Khalaf, M.; Reza F. Investigation of dendrimer structures by means of reverse atomic bond connectivity index. Journal of Discrete Mathematical Sciences and Cryptography 2021, 24, 473-485, http://doi.org/10.1080/09720529.2021.1882161.

26. Nur H.A.M.S.; Mohamad N.H.; Nur B.I. On the zagreb indices of the line graphs of polyphenylene dendrimers. Journal of Discrete Mathematical Sciences and Cryptography 2020, 23,1239-1252, http://doi.org/10.1080/09720529.2020.1822041

27. Khalaf, A.J.M.; Javed, A.; Jamil, M. K.; Alaeiyan, M.; Reza F.M. Topological properties of four types of porphyrin dendrimers. Proyecciones 2020, 39, 979-993, https://doi.org/10.22199/issn.0717-6279-2020-040061.

28. Iqbal, Z.; Aslam, A.; Ishaq, M.; Gao, W. The Edge Versions of Degree-Based Topological Descriptors of Dendrimers. Journal of Cluster Science 2020, 31, 445-452, https://doi.org/10.1007/s10876-019-01658-w.

29. Pattabiraman, K.; Santhakumar, A.; Satheeshkumar, G. Degree based descriptors of certain classes of dendrimer graphs. Materials Today: Proceedings 2021, 42, 1258-1261, https://doi.org/10.1016/j.matpr.2020.12.881.

30. Buragohain, J.; Deka, B.; Bharali, A. A generalized ISI index of some chemical structures. Journal of Molecular Structure 2020, 1208, 127843, https://doi.org/10.1016/j.molstruc.2020.127843.

31. Gao, W.; Iqbal, Z.; Jaleel, A.; Aslam, A.; Ishaq, M.; Aamir, M. Computing entire Zagreb indices of some dendrimer structures. Main Group Metal Chemistry 2020, 43, 229-236, https://doi.org/10.1515/mgmc-20200027.

32. Gao, W.; Akhter, S.; Iqbal, Z.; Qasim, M.; Aslam, A. The Topological Aspects of Phthalocyanines and $\begin{array}{lllll}\text { Porphyrins } \quad \text { Dendrimers. } & \text { IEEE } & \text { Access }\end{array}$ https://doi.org/10.1109/ACCESS.2020.3023658.

33. Vignesh R.; Kalyani D.Neighbourhood Degree - Based Topological Indices of Graphene Structure. Biointerface Research in Applied Chemistry2021, 11, 13681-13694, https://doi.org/10.33263/BRIAC115.1368113694.

34. Sourav M.; Nilanjan D.; Anita P. On some New Neighbourhood Degree Based Indices.Acta Chemica Iasi 2019, 27, 1, 31-46, https://doi.org/10.2478/achi-2019-0003.

35. Sourav M.; Nilanjan D.; Anita P. Topological properties of Graphene using some novel neighborhood degree-based topological indices.International Journal of Mathematics for Industry 2019, 11, 1950006, http://doi.org/10.1142/S2661335219500060.

36. Mondal, S.; De, N.; Pal, A. On Some New Neighborhood Degree-Based Indices for Some Oxide and Silicate Networks. J Multidisciplinary Scientific Journal 2019, 2, 384-409, https://doi.org/10.3390/j2030026.

37. Shanmukha, M.C.; Basavarajappa, N.S.; Usha, A.; Shilpa, K.C. Novel neighborhood redefined first and second Zagreb indices on carborundum structures. Journal of Applied Mathematics and Computing 2021, 66, 263-276, https://doi.org/10.1007/s12190-020-01435-3.

38. Mondal, S.; Dey, A.; De, N.; Pal, A. QSPR analysis of some novel neighborhood degree-based topological descriptors. Complex and Intelligent Systems 2021, 7, 977-996, https://doi.org/10.1007/s40747-020-002620 .

39. Syed A. K. K.; Parvez A.; Faizul A. Topological indices and QSPR/QSAR analysis of some antiviral drugs being investigated for the treatment of COVID-19 patients. International Journal of Quantum Chemistry 2021, 121, 1-22, https://doi.org/10.1002/qua.26594.

40. Sourav M.; Nilanjan D.; Anita P. On neighborhood Zagreb index of product graphs. Journal of Molecular Structure 2021, 1223, 129210, https://doi.org/10.1016/j.molstruc.2020.129210. 